\title{
Cambio climático desde el enfoque de las mujeres rurales en México
}

\section{Climate change from the approach of rural women in Mexico}

\author{
Verónica Gutiérrez Villalpando *, Benito Salvatierra Izaba², Beatriz Martínez Corona ${ }^{3}$ \\ ${ }^{1}$ Consejo Nacional de Ciencia y Tecnología. Comisionada en el Colegio de Postgraduados, Campus Puebla. Blvd. Forja- \\ dores de Puebla 205, Ampliación Momoxpan, CP 72760. Puebla, Puebla, México. \\ 凶bioveros@hotmail.com,vgutiérrez@conacyt.mx \\ D. http://orcid.org/0000-0001-7190-6449 \\ ${ }^{2}$ El Colegio de la Frontera Sur, Unidad San Cristóbal. Carretera Panamericana y Periférico Sur s/n Barrio María Auxilia- \\ dora. CP 29290. San Cristóbal de Las Casas, Chiapas, México. \\ 凶bsalvati@ecosur.mx \\ http://orcid.org/0000-0001-5950-7840 \\ ${ }^{3}$ Colegio de Postgraduados, Campus Puebla. Blvd. Forjadores de Puebla 205, Ampliación Momoxpan, CP 72760. Puebla, \\ 凶Puebla, México. beatrizm@colpos.mx \\ http://orcid.org/0000-0002-0745-4270
}

\section{Resumen}

Se propone un índice de sensación-observación-percepción del cambio climático (ISOP-CC) como variable de respuesta a partir de cinco indicadores (precipitaciones, temperaturas, plagas, vientos y heladas), con el objetivo de medir las reacciones culturales femeninas ante dichos eventos atmosféricos; a las respuestas se les asignaron valores que oscilan entre cero y cien por ciento. El estudio se llevó a cabo en tres localidades del municipio de San Salvador el Verde, ubicadas en la cuenca alta del río Atoyac, en la región conocida como Sierra Nevada de Puebla, México. Entre las variables independientes con impactos en el ISOP-CC se encuentran el gradiente altitudinal relacionado con el tipo de ecosistema y la cobertura forestal, los perjuicios por el cambio climático, la tenencia de la tierra, las actividades económicas del grupo doméstico, la cobertura forestal, las variables sociales y agroproductivas. Se concluye que las mujeres rurales tienen ISOP-CC diferenciales según la altitud de su asentamiento.

Palabras clave: Mujeres rurales, percepciones, cambio climático, gradiente altitudinal, enfoque cuantitativo.

\begin{abstract}
An index of sensation-observation-perception of climate change (ISOP-CC) is proposed as a response variable based on five indicators (rainfall, temperatures, plagues, winds and frosts), with the aim of measuring female cultural reactions to these atmospheric events. Responses were valued from zero to one hundred percent. The study was carried out in three localities of the Municipality of San Salvador el Verde, located in the upper basin of the Atoyac River, in the region known as Sierra Nevada de Puebla, Mexico. Among the independent variables with impacts on the ISOP-CC are the altitudinal gradient related to the type of ecosystem and forest cover, damages due to climate change, land tenure, economic activities of the domestic group, forest cover, social and agricultural productive variables. It is concluded that rural women have differential ISOP-CC according to the altitude of their settlement.
\end{abstract}

Keywords: Rural women, perception, climate change, altitudinal gradient, quantitative approach. 


\section{Introducción}

Se argumenta que la constitución de indicadores tiende a reproducir un esquema positivista que coloca a las dimensiones sociales como mero componente instrumental, cuyo fin es evaluar el impacto del cambio climático en las colectividades humanas. La cuantificación tiene por destino ser insumo para robustecer procesos adaptativos, gestivos y administrativos destinados a grupos, localidades, regiones o países prioritarios. Sin embargo, se critica que éstos subvaloran las sensaciones, observaciones y percepciones derivadas de los conocimientos locales, que pueden ser clave si realimentan las políticas para mitigar el cambio climático (Demeritt, 2001; Forsyth, 2003).

En este estudio consideramos que la construcción social de los conocimientos sobre el entorno (sensaciones, observaciones y percepciones) devienen de relaciones sociales, económicas y políticas que median la interacción entre las personas y el ambiente (Dietz et al., 2013). La intención no es acceder a las definiciones locales sobre los cambios en eventos ambientales, sino en la forma en que los sienten, observan y perciben. Por ello, el objetivo de este estudio fue acceder a los niveles de sensación, observación y percepción de las mujeres en torno al cambio climático, a partir de cinco indicadores (precipitaciones, temperaturas, plagas, vientos $\mathrm{y}$ heladas) que puedan aproximarnos a una detección experiencial sobre la dinámica ambiental en el área.

Las percepciones son dinámicas, se presentan de manera diferencial dependiendo de atributos biofísicos cuantificables. Dada la complejidad en la vinculación de ecosistemas y las mujeres rurales resulta una propuesta innovadora, centrada en la visión local para sentir, observar y percibir eventos asociados al cambio climático.

\subsection{Marco referencial sobre sensación-observa- ción-percepción}

Bajo ciertas circunstancias, la resultante del procesamiento de la información percibida por las personas se da de forma no consciente, es decir, el observador considera que no ha detectado o experimentado cierto aspecto de su entorno vital. De hecho, parte importante de los hechos visuales (excepto el reconocimiento, connaturalmente "consciente") tiene lugar fuera de la conciencia del sujeto (Aznar-Casanova, 2018). La percepción es considerada como el conjunto aditivo de niveles de acercamiento a las partes de un objeto considerado como una totalidad; en este sentido, se ubican tres niveles de aproximación hacia el mismo, a manera de continuo (Aznar-Casanova, 2018), como se observa en la Figura 1.

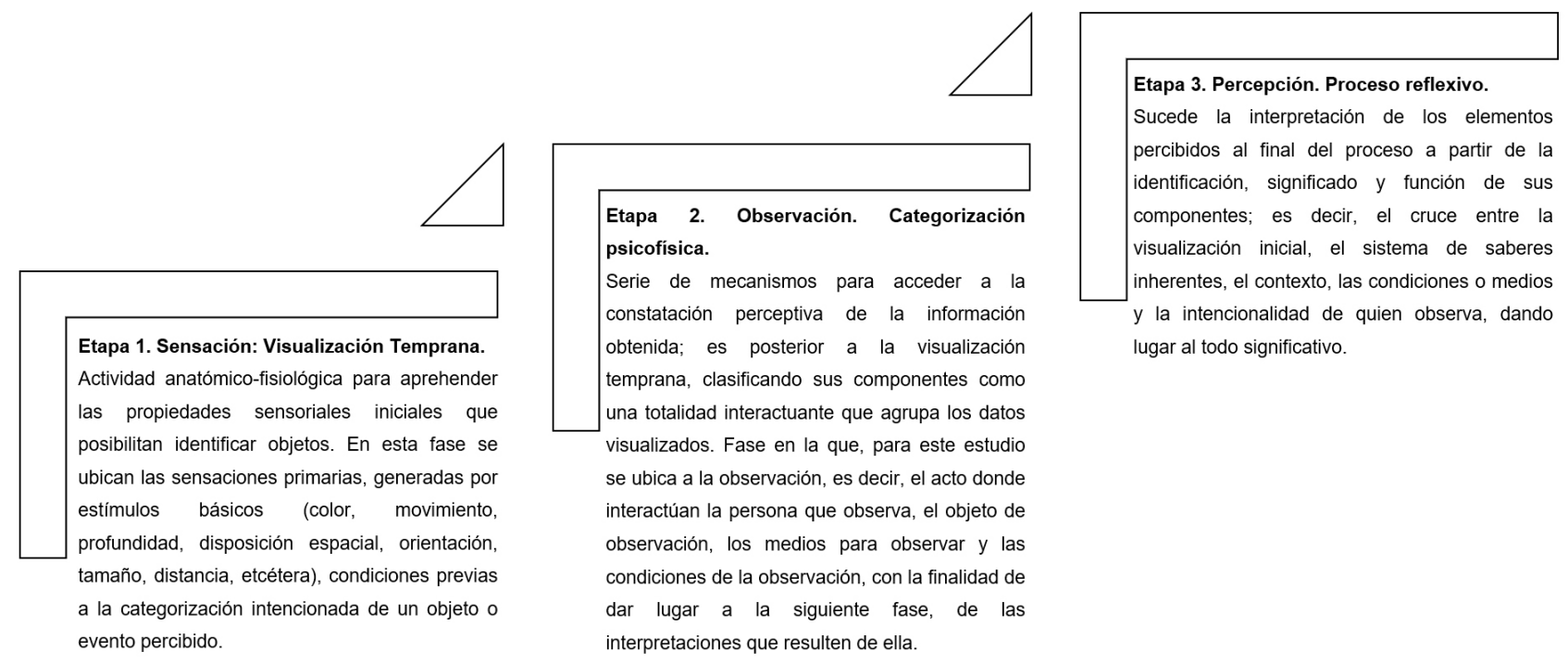

Figura 1. Niveles teóricos en el proceso de acercamiento sensación-observación-percepción. Elaboración a partir de Aznar-Casanova (2018).

En el reconocimiento perceptivo existe una correspondencia entre el procesamiento de la informa- ción visual obtenida, más el respectivo conocimiento almacenado a largo plazo sobre la caracterización 
del entorno, para que, finalmente, se lleve a cabo el análisis que dé lugar a la percepción. Es un ejercicio reflexivo en el que se interseca la sensación primaria, con su categorización, misma que, a su vez, es filtrada con base en la información conceptual y experiencial almacenada, para dar lugar a la percepción reflexiva del cambio climático, no como un evento ambiental azaroso, sino como la concatenación de eventos sentidos, categorizados y, finalmente, evaluados (Aznar-Casanova, 2018). A continuación, en la Tabla 1 se esquematiza el procedimiento de medición referido al índice de sensación-observación-percepción.

Tabla 1. Índice de sensación-observación-percepción.

\begin{tabular}{|c|c|c|c|}
\hline Índice & Sensación & Observación & Percepción \\
\hline \multirow{2}{*}{$\begin{array}{l}\text { Indicadores del cambio } \\
\text { climático }\end{array}$} & \multicolumn{3}{|c|}{$\begin{array}{l}\text { Sí ha sentido, observado o percibido más lluvia + más calor + más plagas + más vientos + más } \\
\text { heladas }=1\end{array}$} \\
\hline & \multicolumn{3}{|c|}{$\begin{array}{c}\text { No ha sentido, observado o percibido más lluvia }+ \text { más calor }+ \text { más plagas }+ \text { más vientos }+ \text { más } \\
\text { heladas }=0\end{array}$} \\
\hline
\end{tabular}

Al final del proceso ocurre la percepción consciente de las distintas características y aspectos de los diversos objetos y entidades que, para este estudio, se tienen sobre el cambio climático en tres localidades del municipio de San Salvador el Verde, ubicadas en la cuenca alta del río Atoyac, en la región conocida como Sierra Nevada de Puebla, México. Líneas adelante se detalla el proceso de "Construcción del índice de sensación-observación-percepción del cambio climático".

Las mujeres poseen un cúmulo de datos procedentes de su sistema de conocimientos local, asociado al marco de creencias, eventos del contexto y destrezas adquiridas, como parte del ámbito socioeconómico y cultural de cada una, dando paso a la valoración de los sucesos (Ruiz, 2014). Por ello, se considera indispensable el conocimiento del presente índice, dado que hoy en día se están presentando constantes variaciones en el clima, y más allá de recetar pautas estandarizadas para su mitigación, lo que se busca es proporcionar insumos locales para que, por una parte, los tomadores de decisiones perciban el cambio climático a partir de la mirada de las mujeres rurales y en contextos económicos depauperados; y por la otra, se dialoguen estrategias en donde las iniciativas tengan sentido para las poblaciones directamente vinculadas con el ambiente, en función de cierta gama de apreciaciones, y no solo sean receptáculos de programas generalizados. Es decir, generar igualdad de oportunidades a partir de la diversidad.

\subsection{Antecedentes en el uso de los indicadores e in- dices ambientales}

Desde hace algunas décadas, en el ámbito ambiental se han desarrollado indicadores para enten- der, describir y analizar distintos fenómenos como el clima, la pérdida de suelos y el riesgo de especies, entre muchos otros (NOM-161-SEMARNAT-2011). Sin embargo, no existe una definición única del concepto y éste varía de acuerdo con los objetivos específicos que se persiguen. Una de las definiciones más comunes y aceptadas proviene de la Organización para la Cooperación y el Desarrollo Económico [OECD] (1993) que define un indicador ambiental como un parámetro o valor derivado de parámetros que proporciona información para describir el estado de un fenómeno, ambiente o área con un significado que va más allá del directamente asociado con el valor del parámetro en sí mismo. La OCDE, desde hace años utiliza un conjunto de indicadores como información base para realizar evaluaciones periódicas del desempeño ambiental de los diferentes países que integran la organización (NOM-161-SEMARNAT-2011).

Los indicadores se caracterizan por expresarse como una señal física o numérica en escala del $0-1$ o de $0-100 \%$, estar inscritos en un marco teórico asociado al desarrollo sustentable o al problema que se pretenda estudiar, generar información útil para realizar comparativos y enfocarse en evaluar algún aspecto específico (Ibáñez Pérez, 2012; Cegarra, 2019). También, pueden clasificarse en función de diferentes criterios, con base en el sentido teórico y la obtención de datos para su elaboración (Ibáñez Pérez, 2012).

Existen dos tipos de indicadores: objetivos y subjetivos. Los indicadores objetivos (duros), se fundamentan en evidencias externas independientes del informante (ejemplo: calidad del agua, emisión 
de partículas contaminantes), suponiendo que los métodos de captación y procesamiento de la información son objetivos. En cambio, los indicadores subjetivos (alternos), reflejan percepciones y opiniones de la población respecto a su situación (ejemplo: percepción de la participación social).

Tanto Perevochtchikova (2012) como el Consejo Nacional de Evaluación de la Política de Desarrollo Social (CONEVAL, 2019) señalan que los indicadores son de enorme utilidad en la planeación y toma de decisiones, tanto en el ámbito local como regional y nacional (Donnelly et al., 2006; Consejo Territorial de Planeación Distrital, 2020). Sin embargo, éstos presentan retos importantes entre los que destacan los siguientes: a) restricciones institucionales: se requiere un mayor trabajo en el desarrollo de metodologías que aborden temas estadísticos e indicadores, así como el entrenamiento en la construcción de indicadores (Cegarra, 2019).

También existen limitaciones en los recursos económicos, humanos e infraestructura; dificultades en la agregación y la comparación de datos; falta de sistemas de compilación de información y relevancia en ciertos temas, y b) restricciones técnicas: falta de capacitación en las metodologías relacionadas con los indicadores de desarrollo sustentable; datos básicos o de estadísticas en términos de calidad y cantidad (Cegarra, 2019; CONEVAL, 2019).

Asimismo, las diferentes definiciones implican las posibilidades de una mala interpretación; cobertura deficiente de redes de monitoreo, lo que resulta en vacíos de datos en las series de tiempo y diferentes periodos reportados que indican dificultades técnicas y conceptuales de medición, lo que complica su comparación (Shah, 2004; CONEVAL, 2019; Consejo Territorial de Planeación Distrital, 2020).

Frecuentemente, se utilizan las palabras "parámetro" e "índice" como sinónimos de indicador, sin embargo, no tienen el mismo significado. Un parámetro se define como cualquier propiedad que es medida u observada, mientras que un índice designa a un conjunto agregado o ponderado de cuantificaciones o indicadores (Consejo Territorial de Planeación Distrital, 2020). Cuando se utiliza un valor para describir la situación de un tema, frecuentemente ocurre que no refleja bien la condición del sistema o la simplifica, de manera que resulta inútil para la toma de decisiones. Por ello, a menudo se recurre a la elaboración de índices o conjuntos de indicadores (Cegarra, 2019; CONEVAL, 2019).
En temas complejos, como el ambiental, la elaboración y utilización de índices es muy atractiva porque permiten una visión general de cierta situación. Los índices se componen por diferentes indicadores que representan un reto para regiones con gran superficie territorial, o con poca información para determinar sus condiciones de daño o conservación en un momento dado (Cegarra, 2019). Algunos de los índices más conocidos que tratan de evaluar la sustentabilidad ambiental son el índice del Planeta Viviente (Living Planet Index), la Huella Ecológica (Ecological Footprint) y el Índice de Sustentabilidad Ambiental (Environmental Sustainability Index) (NOM-161-SEMARNAT-2011; Instituto Nacional de Ecología y Cambio Climático [INECC], 2020).

\subsection{Enfoques del abordaje en las investigaciones sobre cambio climático}

En este estudio se parte del concepto de que el clima es una construcción cultural que se elabora a partir de procesos materiales y simbólicos, y que denota aspectos culturales, espaciales e históricos (VanderMolen, 2011). El planteamiento del problema de investigación se aborda desde la perspectiva de que la vulnerabilidad al cambio climático es diferencial y depende del gradiente altitudinal de las comunidades. Con base en la teoría ecosistémica y el enfoque de cuencas (Semarnat y Centro de Educación y Capacitación para el Desarrollo Sustentable [CECADESU], 2016, pp. 19-28), a mayor gradiente altitudinal, menor es la vulnerabilidad ante el cambio climático (Manzanilla-Quiñones, 2019).

La vulnerabilidad al cambio climático, a su vez, está mediada por el capital natural, el manejo de la producción agrícola y las asignaciones de género, asumiendo que las mujeres al estar más tiempo en las comunidades de origen son más sensibles que otros actores sociales (autoridades, hombres, instituciones) para detectar las tendencias de las variables principales aleatorias del cambio climático (Women's Environmental Network, 2010; Ruiz, 2014). Esta comprensión de la vulnerabilidad socio ambiental es clara cuando se analizan las percepciones desde el enfoque de cuencas, el cual permite cuantificar e identificar los impactos acumulados de las actividades humanas a lo largo de la red de arroyos y ríos, que afectan positiva o negativamente la calidad y cantidad de agua (Martínez-Austria, 2012; Orozco et al., 2018; Soares et al., 2018).

En este estudio se analiza si las sensaciones, observaciones y percepciones de las mujeres rura- 
les pueden contribuir a identificar cambios en cinco indicadores de cambio climático: 1luvia, heladas, viento, plagas y calor. Con estos elementos ambientales se construyó un índice de sensación-observación-percepción del cambio climático (ISOP-CC), tomando a esta triada como un fenómeno social.

Las investigaciones que abordan la temática del cambio climático se han enfocado en dos vertientes principales:

i. Ciencias naturales: el cambio climático es una descripción estadística del tiempo atmosférico en términos de los valores medios y de la variabilidad de las magnitudes correspondientes durante periodos que pueden abarcar desde meses hasta millares o millones de años. El promedio del periodo habitual es de 30 años (Grupo Intergubernamental de Expertos sobre el Cambio Climático [IPCC], 2007). El conocimiento científico ha puntualizado y demostrado que las principales manifestaciones del cambio climático son la ocurrencia o intensidad de los fenómenos de variabilidad climática ocasionados por el ciclo El Niño Oscilación del Sur (ENOS) (Pinilla et al., 2012). Con estos datos se ha logrado la construcción de escenarios futuros, que en general han sido poco alentadores. Las instituciones que han medido las variables climatológicas se han basado en datos duros asociados a temperatura y precipitación. Los valores provienen de las diferentes estaciones meteorológicas y están relacionados con los cambios de uso de suelo, cobertura vegetal, enfermedades, impactos agrícolas y pecuarios, entre otros (Solís \& Salvatierra, 2013).

ii. Ciencias sociales: el cambio climático es el resultado de la forma en que los individuos sienten, observan, perciben, se apropian e interpretan los fenómenos meteorológicos y climáticos que ocurren a su alrededor (Soares et al., 2018). Asimismo, los gobiernos tienden a confiar en la investigación científica sobre el cambio climático, pero a menudo ignorando la dinámica en los sistemas socioeconómicos regionales y locales, al predecir los impactos del cambio climático y al legislar estrategias y políticas de adaptación a nivel global (Adger \& Kelly, 1999; Duarte, 2014; Fuentes y Brugués, 2017; Roth, 2019; Revuelta, 2019).

De acuerdo con Ulloa (2013) se han emprendido acciones a nivel local en las que se generali- zan las estrategias de adaptación sin considerar los contextos locales específicos y las desigualdades de género. Algunas investigaciones previas indican que las personas locales son más capaces que otros de percibir y adaptarse a los aspectos de la variabilidad y el cambio climático, basados en la observación personal (Deressa et al., 2009; Spence et al., 2011; Howe et al., 2013; Mayo, 2019). Las observaciones de los residentes locales están integradas en la cultura local (Ruiz, 2014).

La noción de clima y sus variaciones entre la población en general se asocia a construcciones y representaciones sociales, sensaciones, observaciones, percepciones, saberes y formas coloquiales de referirse al término que repercuten en la forma en que se organizan y participan hombres y mujeres para hacer frente a este fenómeno (Revuelta, 2019).

Cabe aclarar que nociones como la del cambio climático, su variabilidad, así como los eventos extremos implican dinámicas ambientales diferentes. Por variabilidad climática se hace referencia a valores por encima o debajo de su valor promedio; mientras que el cambio climático implica una tendencia (mayor o menor) en alteraciones que persisten a largo plazo en las condiciones climáticas. Los eventos extremos hacen alusión a sucesos específicos en temperatura, heladas, lluvias, sequía, que provocan serios daños por su magnitud.

Asimismo, se analizan las percepciones sociales del ambiente que se definen como el conjunto de comprensiones y sensibilidades de una sociedad, integrada por hombres y mujeres, sobre su ambiente natural y surgen a través de un proceso de asociación de significados a los elementos del entorno y a sus cursos de transformación y deterioro (Lazos \& Pare, 2016; Cegarra, 2019), además de las asignaciones de género (Lazos \& Pare, 2016). Éstas, constituyen sistemas cognitivos en los que es posible reconocer la presencia de opiniones, creencias, valores y normas que tienen las personas sobre el ambiente natural, las cuales van a determinar la orientación de actitudes positivas o negativas con respecto a la conservación de la naturaleza (Bertoni \& López, 2010; Ruiz, 2014).

Conocer e incluir las percepciones ambientales de los actores es clave para la formulación de propuestas de desarrollo sostenible (Millán, 2004; Cegarra, 2019), porque permiten dirigir proyectos o esfuerzos para el manejo de los recursos naturales, acorde con las referencias locales (Revuelta, 2019). 
Esto puede verse reflejado en procesos más incluyentes y equitativos, que son esenciales para llevar a cabo un manejo sostenible de los recursos naturales y mantener el bienestar de las comunidades rurales (Infante \& Arce, 2015; CONEVAL, 2019)

Desde las ciencias sociales, las respuestas individuales e institucionales frente al cambio climático han recibido una atención especial (Oltra et al., 2009). En este sentido, la generación del conocimiento sobre las percepciones del cambio climático con base en el análisis crítico se ha abordado en la mayoría de los estudios a partir de la metodología cualitativa (Soares et al., 2018; CONEVAL, 2019).

Sin embargo, también se requiere que sean documentados con el rigor metodológico estadístico, y con ello dar sustento a la reconceptualización social del fenómeno, sus manifestaciones y afectaciones en territorios concretos, como es el caso de las comunidades rurales de la Sierra Nevada de Puebla. Dichas percepciones están mediadas por factores fisiográficos, históricos, políticos, económicos, culturales, ambientales y de género.

El estudio de estas percepciones desde el enfoque cuantitativo como el que se realiza en este estudio permitirá un mejor entendimiento del fenómeno por parte de los tomadores de decisiones y manejo adecuado de esta problemática por parte de los campesinos y campesinas, con miras a la generación de estrategias locales de adaptación a las variantes climáticas y a fenómenos meteorológicos extremos generados por el cambio climático (Cegarra, 2019).

A partir de esta aproximación cuantitativa, se presentan los resultados de una encuesta realizada a 240 mujeres de tres comunidades del municipio de San Salvador el Verde perteneciente al estado de Puebla, México, que fueron beneficiarias del extinto Programa Prospera, una de las siete líneas estratégicas a cargo de la Secretaría de Desarrollo Social (Sedesol), en la administración gubernamental de 2012-2018 en México. Actualmente, en el sexenio 2018-2024, dicha política pública se denomina Secretaría de Bienestar (Bienestar) y se redujo a tres programas.

Las tres comunidades del municipio de San Salvador el Verde están ubicadas en la cuenca alta del río Atoyac, en la región conocida como Sierra Nevada de Puebla. Dada su posición geográfica se les consideró adecuadas para el presente estudio, considerando como objetivo analizar si existe una interacción entre el gradiente altitudinal, y la interrelación con el ecosistema, manifiesta a partir de las estrategias de producción, mismas que son la expresión de una cultura. La interacción entre los criterios mencionados (altitud+ecosistema+producción+prácticas culturales) posibilita explicar la varianza de los indicadores de cambio climático percibidos por las mujeres rurales entrevistadas.

El componente social y sobre mujeres en este estudio permitió responder las siguientes interrogantes: 1) ¿Existen diferencias en cómo perciben las mujeres rurales locales la variabilidad en el clima en los diferentes niveles altitudinales?; 2) ¿Existe alguna asociación entre el índice de percepción observación y sensación del cambio climático y los factores socioeconómicos como la educación, las actividades económicas primaria y secundaria de las entrevistadas, la actividad económica del esposo, el perjuicio sufrido por el cambio climático, la tenencia de la tierra y los ingresos económicos del grupo doméstico?; y 3) ¿las mujeres rurales perciben, observan o sienten los efectos de la variabilidad climática en su bienestar?

\section{Metodología}

\subsection{Generalidades del territorio, su clima y medios de vida}

El municipio de San Salvador el Verde forma parte de la Sierra Nevada, que a su vez se incluye en el sistema volcánico transversal, con una altitud de $2.500 \mathrm{~m}$ s.n.m. En este municipio se presenta la transición de los climas templados del valle de Puebla a los semifríos de las partes bajas de la Sierra Nevada; se identifican dos climas: clima templado subhúmedo y semifrío subhúmedo con lluvias en verano. La temperatura media anual oscila entre 12 y $18^{\circ} \mathrm{C}$ y entre -3 y $18^{\circ} \mathrm{C}$ en el mes más frío, además, existe precipitación invernal. Respecto a su hidrografía, pertenece a la cuenca del río Atoyac, una de las cuencas más importante del estado, pero a su vez una de las más contaminadas, sobre todo por la descarga de compuestos químicos que vierten las fábricas de textiles. Su relieve es accidentado con praderas de alta montaña y gran diversidad de suelos (González-Pérez et al., 2019).

\subsection{Universo de estudio}

El universo de estudio comprendió tres localidades ubicadas en el municipio de San Salvador el Verde, cuyas coordenadas geográficas, altitud y po- 
blación total se recogen en la Tabla 2. En la Figura 2 observamos los gradientes altitudinales presentes en el municipio de San Salvador el Verde. Las comunidades de estudio son 13a, 13b y $13 \mathrm{~d}$.

Tabla 2. Localidades estudiadas y su altitud en el municipio de San Salvador El Verde.

\begin{tabular}{llcr}
\hline \multicolumn{1}{c}{ Comunidad } & \multicolumn{1}{c}{ Coordenadas } & $\begin{array}{c}\text { Altitud } \\
\text { (m s.n.m.) }\end{array}$ & $\begin{array}{c}\text { Población total } \\
\text { (hab.) }\end{array}$ \\
\hline San Andrés Hueyacatitla & $\begin{array}{l}\text { Latitud } 19^{\circ} 15^{\prime} 19^{\prime \prime} \mathrm{N} \\
\text { Longitud } 09^{\circ} 86^{\prime} 156^{\prime \prime} \mathrm{O}\end{array}$ & 2.471 & 4.529 \\
Cabecera municipal de San Salvador el Verde & $\begin{array}{l}\text { Latitud } 19^{\circ} 16^{\prime} 15^{\prime \prime} \mathrm{N} \\
\text { Longitud } 09^{\circ} 83^{\prime} 053^{\prime \prime} \mathrm{O}\end{array}$ & 2.401 & 2.768 \\
& $\begin{array}{l}\text { Latitud } 19^{\circ} 55^{\prime}, 52^{\prime \prime} \mathrm{N} \\
\text { Longitud } 09^{\circ} 83^{\prime} 001^{\prime \prime} \mathrm{O}\end{array}$ & 2.375 & 2.521 \\
\hline
\end{tabular}

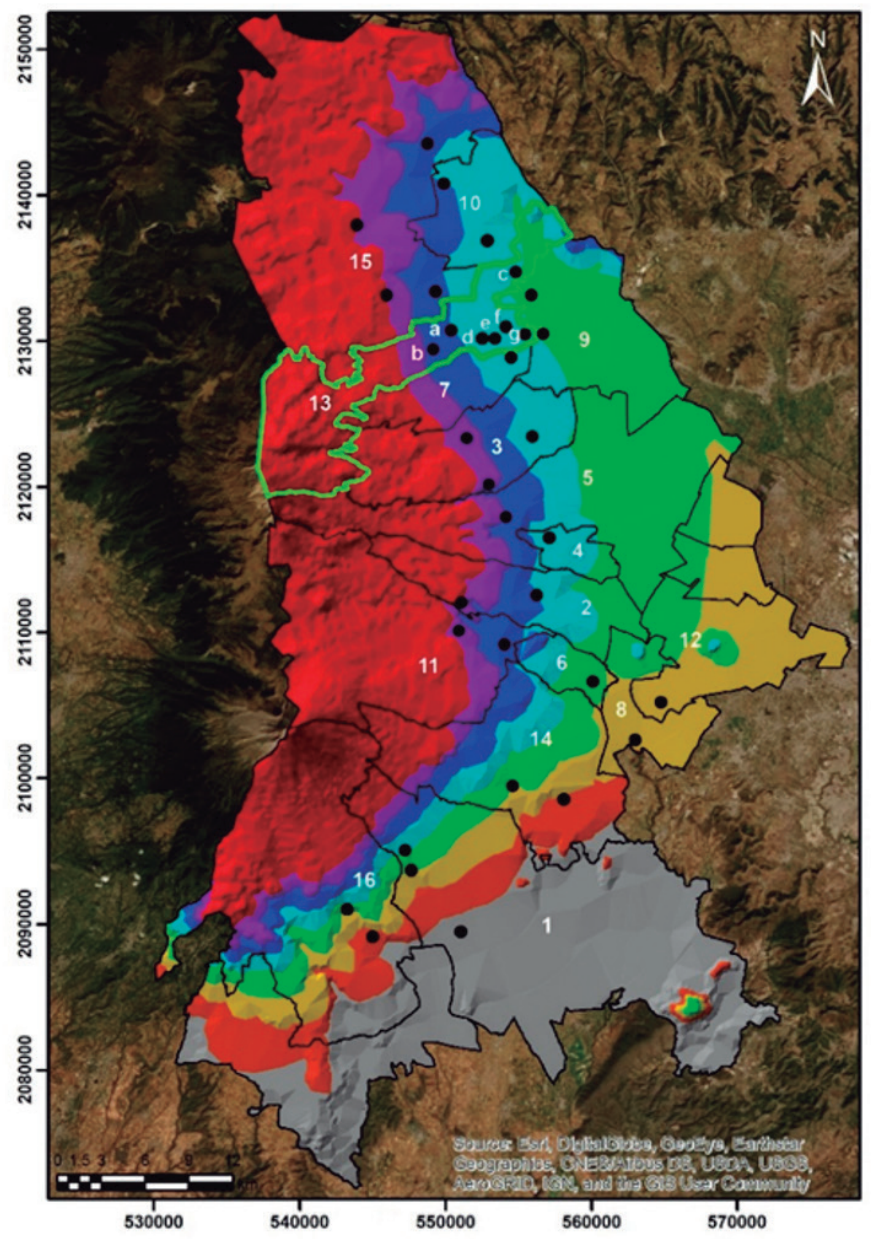

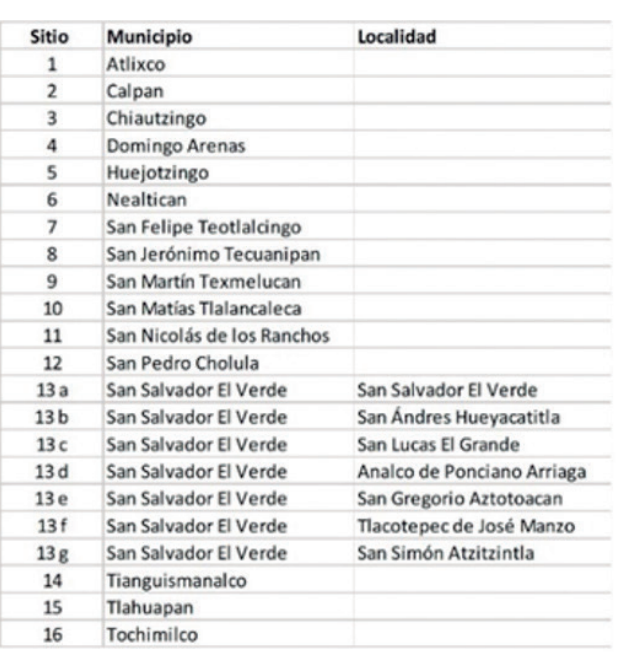

- Núcleos ejidales

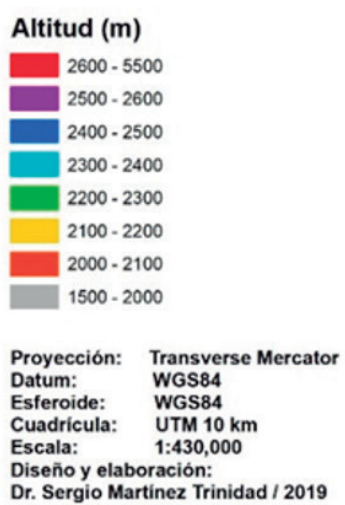

Figura 2. Localidades estudiadas y su altitud en el municipio de San Salvador el Verde.

En este estudio, las comunidades se clasificaron con base en su ubicación y gradiente altitudinal en comunidad alta, media y baja, respectivamente, todas pertenecen a la cuenca alta del río Atoyac. La información se obtuvo por observación participante, geolocalización, la aplicación de un cuestionario estructurado y precodificado con un total de 40 preguntas divididas en 5 secciones y entrevistas a las personas clave, todas mujeres. 


\subsection{Caracterización de la zona de estudio}

Los cultivos de temporal que predominan en estas comunidades son el maíz y el frijol para autoconsumo. Los campesinos(as) que cuentan con sistema de riego también se dedican al cultivo de frutales como tejocote, manzana, pera, ciruela y durazno que tienen una alta demanda en el mercado nacional e internacional. Cabe mencionar, como parte de la agricultura protegida, el incremento del cultivo de flores y hortalizas en invernadero que son actividades recientes y surgieron por la necesidad de diversificarse para generar nuevos ingresos económicos y que en los últimos veinte años ha aumentado su superficie, desplazando a la agricultura convencional, principalmente para fines comerciales. Asimismo, se observa ganadería extensiva en una escala menor.

Es importante mencionar que la comunidad de San Andrés Hueyacatitla, ubicada en la parte más alta, es la que cuenta con mayor número de habitantes, y por su altitud tiene más cercanía con el bosque (capital natural). En esta localidad existe un manejo forestal maderable que, de acuerdo con su nivel de desarrollo de empresa forestal comunitaria en México, corresponde a un tipo de productor primario, donde los dueños o poseedores de recursos forestales que tienen programas de manejo y permisos de extracción de productos maderables, participan en alguna fase de la cosecha forestal, así como en algún aspecto del transporte, venta y/o transformación primaria (Hodgdon et al., 2013). Además, los ejidatarios y ejidatarias de esta comunidad también reciben pago por servicios ambientales (PSA).

Un actor institucional clave con presencia y reconocimiento en este ejido es la Comisión Nacional Forestal (Conafor), cuya operatividad se basa en dos componentes: el Programa Estratégico Forestal 2025 (2001) y la Ley de Desarrollo Forestal Sustentable (2003).

Asimismo, los ejidatarios y ejidatarias de esta comunidad practican la acuacultura mediante el cultivo de trucha arcoíris (Oncorhynchus mykiss) en pequeños estanques de tierra. Recientemente, también han incursionado en el ecoturismo a través del avistamiento de luciérnagas (Macrolampis palaciosi), donde ofrecen un recorrido general, zona de contemplación para visitantes con alguna discapacidad, tirolesa, trucha fresca al gusto servida en su restaurante, actividades de remo en un pequeño lago alimentado por los manantiales provenientes del volcán Iztaccíhuatl y hospedaje para los turistas que así lo requieran.

\subsection{Diseño muestral}

El tamaño muestral se estimó en 269 hogares (Cochran, 2000), de los cuales se obtuvo información completa de 239 mujeres entrevistadas, que eran o las jefas de hogar o las esposas del jefe de familia, una persona por cada grupo doméstico. La muestra estudiada tuvo una tasa de no respuesta del $11,1 \%$.

1) Analco de Ponciano Arriaga (13d), número de muestra $=63$, se ubica en la parte baja, siendo la localidad con menor población (2.521 habitantes), pero con una fábrica de toallas que contamina el arroyo con colorantes y emite olores desagradables, provenientes de los compuestos químicos que se utilizan en su elaboración; muchas veces los cultivos son regados con esta agua contaminada, lo que puede ocasionar riesgos en la salud.

2) Cabecera municipal de San Salvador el Verde (13a), número de muestra $=33$, se ubica en la parte media (2.768 habitantes) y corresponde a la cabecera municipal, con actividad política y económica comercial, ciudad urbanizada; $y$,

3) San Andrés Hueyacatitla (13b), número de muestra $=143$, asentada en la parte alta ( 2.471 m s.n.m.), es la zona rural semidispersa, con población originaria y la más poblada ( $\mathrm{n}=4.529$ habitantes), pero a su vez es la que cuenta con el mayor capital natural constituido por su bosque de pino, manantiales y por la cercanía al volcán Iztaccíhuatl; su actividad económica es el manejo forestal maderable y el cultivo de frutales. Últimamente están dando los primeros pasos en el turismo de naturaleza con el avistamiento de las luciérnagas durante un par de meses al año (junio-julio).

El trabajo se realizó en los meses de noviembre y diciembre de 2017, y revisitas en enero de 2018.

\subsection{Construcción del índice de sensación-observa- ción-percepción del cambio climático (ISOP-CC).}

Con la información obtenida mediante un cuestionario estructurado y precodificado, se construyó un índice de sensación-observación-percepción del cambio climático (ISOP-CC). A nivel local se siente, observa y percibe de manera diferente el impacto del cambio climático en torno a las variaciones de los indicadores de precipitación, temperatura, plagas, viento y heladas, relacionadas directamente con factores fisiográficos como el gradiente altitudinal de la comu- 
nidad, factores ambientales como la cobertura vegetal y sociales como el perjuicio sufrido por el cambio climático y la tenencia de la tierra del grupo doméstico.

Para analizar la información de este artículo se construyó una base de datos con 15 variables, fisiográficas, socioeconómicas y ambientales relacionadas con el cambio climático: las variables independientes fueron: 1) comunidad y altitud, 2) número de años de estudios escolarizados, 3) actividad económica primaria, 4) actividad económica secundaria, 5) actividad económica principal del esposo, 6) daños experimentados en la producción agrícola asociados con el cambio climático, 7) ingreso per cápita por persona, 8) tenencia de la tierra del grupo doméstico, y 9) número de años que han transcurrido desde que percibió por primera vez el cambio climático (aproximadamente de 5 a 20 años, desde 1997 a 2017).

Las variables o indicadores para elaborar el índice de sensación-observación-percepción del cambio climático (ISOP-CC) fueron los atributos subjetivos y objetivos, derivados de la sensación, observación y percepción del cambio climático por las mujeres entrevistadas, mis- mos que se dirigieron a registrar si han sentido y observado los siguientes elementos meteorológicos con sus respectivos impactos: 10a) lluvias, 10b) heladas, 10c) vientos, 10d) calor y 10e) plagas, versus su percepción de incremento o descenso hacia los mismos.

Para obtener un indicador cuantitativo de los atributos subjetivos y objetivos sobre el cambio climático (ASOCC), se elaboró un cuadro de contingencia de dos por dos para cada elemento del CC (10a, 10b, 10c, 10d y 10e), en donde a cada combinación de respuesta le puede corresponder un valor que va de cero a uno. La suma de la combinación de respuesta podría ir como una función de probabilidad desde cero hasta uno F(ISOP-CC) $=0,0,1,0,2, \ldots, 0,9$ y 1,0 , inclusive con todos los valores intermedios respectivos posibles.

En la Figura 3 se presenta un esquema conceptual de la interacción entre lo observado (sí/no) y la percepción de (incremento/descenso) de la variable principal (VAP) ISOP-CC. El modelo resultante se podría sintetizar así: $\mathrm{F}(\mathrm{ISOP}-\mathrm{CC})=$ sumatoria $(\mathrm{EA} /$ $\mathrm{n}_{\mathrm{ea}}$ ); donde $\mathrm{n}_{\mathrm{ea}}$ es la suma de respuestas correctas con relación a los atributos señalados en la Tabla 3 .

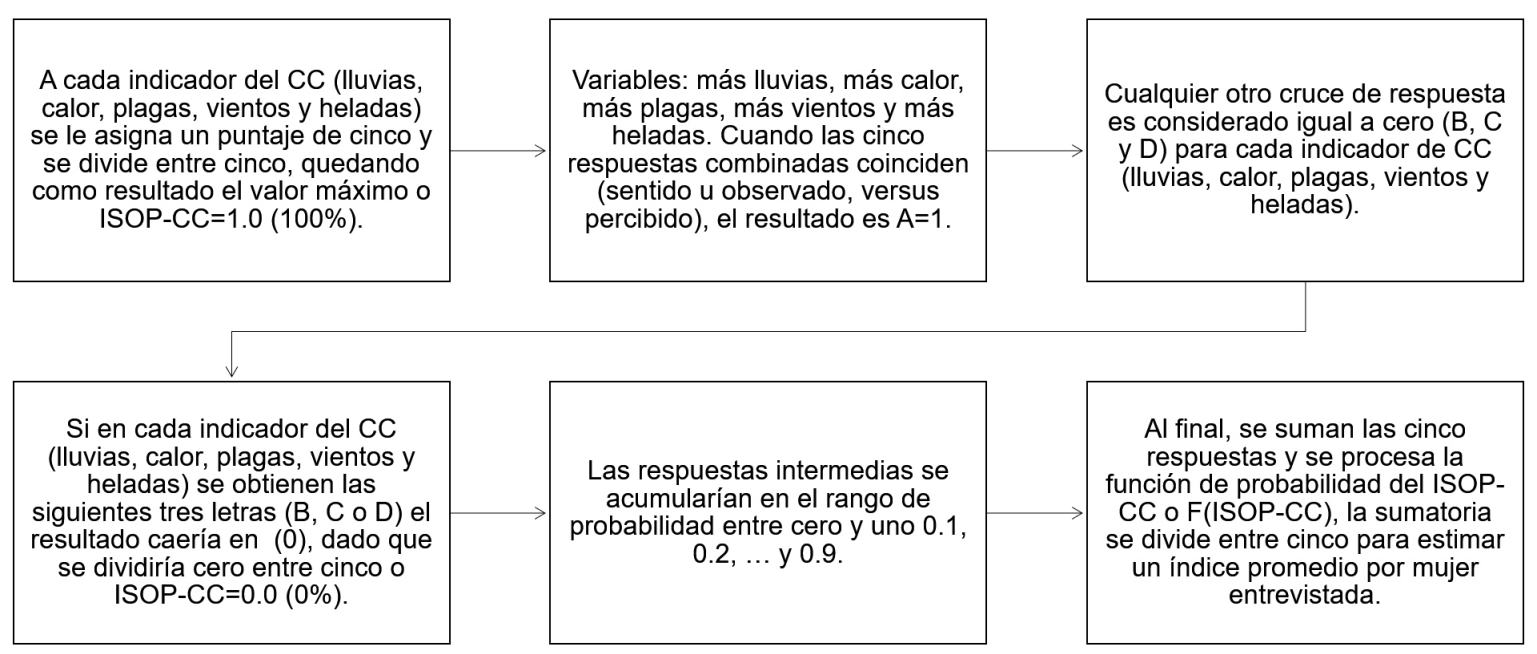

Figura 3. Esbozo procesual para la conformación de la VAP ISOP-CC.

Tabla 3. Delimitación conceptual de la interacción entre lo observado (sí/no) y la percepción de (incremento/descenso) de la VAP ISOP-CC.

\section{Construcción del ISOP-CC}

Indicadores

¿Ha sentido, observado o percibido cambios en los siguientes eventos atmosféricos?

\begin{tabular}{lllllllllll}
\hline Ha percibido & Sí (1) & No $(0)$ & Sí (1) & No (0) & Sí (1) & No (0) & Sí (1) & No (0) & Sí (1) & No (0) \\
Incrementos (+) & A (1) & B (0) & A (1) & B (0) & A (1) & B (0) & A (1) & B (0) & A (1) & B (0) \\
Descensos (-) & C (0) & D (0) & C (0) & D (0) & C (0) & D (0) & C (0) & D (0) & C (0) & D (0) \\
\hline
\end{tabular}


Se compararon las tres comunidades, observándose la menor percepción de cambio climático en San Andrés Hueyacatitla (SAH), que fue clasificada como la comunidad más alta y con más capital natural, que corresponde a la región rural indígena más próxima a la zona menos perturbada y al volcán Iztaccíhuatl (Tabla 2).

\subsection{Indicadores predictores del ISOP-CC}

Los indicadores predictores del ISOP-CC fueron 9: altitud, comunidad 1-3, comunidad 2-3, actividad económica principal y secundaria de la entrevistada, actividad económica principal del esposo, el ingreso per cápita mensual, la tenencia de la tierra por grupo doméstico, años que han transcurrido desde que percibió cambio climático, perjuicios por el cambio climático y años de estudio de la entrevistada.

Para el análisis de datos se realizó una comparación bivariada entre el ISOP-CC y los diferentes indicadores predictores, comparándose por medio de una t de Student y el análisis de varianza de una vía; en los casos de significancia estadística se realizaron pruebas de comparación múltiple de diferencia mínima significativa (DMS).

Para medir la tenencia de la tierra se realizó la pregunta: ¿cuenta con tierras en el grupo doméstico? aunque la entrevistada no fuera la dueña de la tierra. Si la respuesta fue sí $=1$ y si fue no $=0$.

Con los predictores que previamente habían sido sometidos a modelos bivariados se seleccionaron aquellos cuyo valor de error tipo 1 fue menor o igual $(<=) 0,15$ (significativos y marginalmente significativos). Después se utilizó el modelo de regresión lineal general con método de introducción manual de las variables independientes.

Las variables significativas y marginalmente significativas fueron incluidas en un modelo de regresión cuyo resultado es: ISOP-CC $=$ Constante + Comunidad 1-3 + Comunidad 2-3 + perjuicios atribuidos al CC $(1,0)+$ tenencia de la tierra del grupo doméstico $(1,0)$ y tiempo de la primera observación cambio climático (años).

Metodológicamente se validó la confiabilidad del ISOP-CC a través de la comparación de las tendencias percibidas (desde qué año ha observado cambio climático versus la tendencia de medidas de temperatura y lluvias en la estación meteorológica más cercana a la zona de estudio y que corresponde a San Martín Texmelucan, para confirmar la validez externa del índice ISOP-CC (Tabla 4).

Tabla 4. Índice de sensación-observación-percepción del cambio climático.

\begin{tabular}{lcccccc} 
& ISOP-TLL & Vientos & Plagas & Calor & Heladas & Lluvias \\
\hline ISOP-CC & & $0,743^{* *}$ & $0,721^{* *}$ & $0,699^{* *}$ & $0,621^{* *}$ & $0,560^{* *}$ \\
Calor & $0,793^{*}$ & $0,493^{* *}$ & $0,523^{* *}$ & & $0,212^{* *}$ & $0,210^{* *}$ \\
Lluvias & 0,56 & $0,160^{* *}$ & $0,227^{* *}$ & & $0,215^{* *}$ & \\
ISOP-TLL & $0,793^{*}$ & $0,392^{*}$ & $0,457^{* *}$ & $0,712^{* *}$ & 0,273 & 0,836 \\
Percepción CC & & 0,162 & 0,182 & 0,276 & 0,13 & 0,106 \\
\hline
\end{tabular}

\section{Resultados}

\subsection{Indicadores de cambio climático}

Los indicadores de cambio climático con que se construyó el índice de percepción, observación, sensación del cambio climático (ISOP-CC) que tuvieron mayor varianza fueron las heladas, las lluvias y los vientos. Los indicadores de cambio climático con menor varianza fueron calor y plagas. Los predictores del ISOP-CC son la altitud de la comunidad $(\beta=-0,54)$, los perjuicios en la producción agrícola que han experimentado atribuidos al cambio climático $(\beta+)$ y la tenencia de la tierra del grupo doméstico $(\beta+)$ (Tabla 4).

A partir de los años de estudio de las entrevistadas, se observó que éstos no tienen injerencia en una mayor o menor percepción en los elementos indicadores del cambio climático, por lo que podemos afirmar que la percepción, observación y sensación del cambio climático es independiente del 
nivel educativo de las mujeres rurales entrevistadas (Tabla 5). Respecto a los ingresos económicos del grupo doméstico, el ingreso per cápita de los integrantes de cada grupo doméstico, no influye en que las mujeres perciban, observen o sientan más o menos los indicadores del cambio climático. Con relación a la actividad económica primaria y secundaria de las mujeres, no se encontró diferencia estadística significativa en la percepción, observación y sensación del cambio climático entre las mujeres que son agricultoras (campesinas) y las que no lo son. Asimismo, no se encontró diferencia estadística significativa en la percepción, observación y sensación del cambio climático entre las mujeres cuyos esposos son agricultores (campesinos) y las mujeres cuyos esposos no son agricultores o jornaleros. Sin embargo, al comparar el ISOP-CC entre las mujeres que reportaron haber sufrido perjuicios por el cambio climático y las que no, se observa una diferencia de $15 \%$ que representa que existe $23,4 \%$ mayor percepción, observación y sensación del cambio climático entre las que reportan haber tenido perjuicios por el mismo, con respecto a los que reportan que no. El resultado es marginalmente significativo estadísticamente $(\mathrm{t}=16,5 ; \mathrm{gl}=236 ; p$ $=0,09)$ (Tabla 5).

Tabla 5. Análisis de varianza (ANOVA)

\begin{tabular}{|c|c|c|c|c|c|c|c|c|c|c|}
\hline & & $\begin{array}{c}\text { Altitud } \\
\text { (m s.n.m.) }\end{array}$ & $\begin{array}{l}\text { Diferencia } \\
\text { de altitud } \\
\text { (m s.n.m.) }\end{array}$ & n & $\begin{array}{c}\text { X(IC 95\%) } \\
\text { (N/D) }\end{array}$ & GI & $\mathbf{F}$ & $p$ & $\begin{array}{l}\text { COMP } \\
\text { MULT }\end{array}$ & $p$ \\
\hline \multirow[t]{4}{*}{ ISOP_CC } & 1 & 2.375 & & 63 & $0,717(0,658-0,776)$ & $(2,236)$ & & & $1--2$ & 0,552 \\
\hline & 2 & 2.401 & -26 & 33 & $0,678(0,580-0,777)$ & & & & $1--3$ & 0,006 \\
\hline & 3 & 2.771 & -70 & 143 & $0,590(0,5350-645)$ & & 4,253 & 0,015 & $2--3$ & 0,131 \\
\hline & & & -96 & 239 & $0,636(0,596-67)$ & & & & & \\
\hline
\end{tabular}

Prueba $t$

\begin{tabular}{|c|c|c|c|c|c|c|}
\hline \multirow{18}{*}{ ISOP_CC } & indtierra & $\mathbf{n}$ & $\mathbf{X}(\mathbf{E E})$ & $t(g l)$ & $p$ & DIFF(IC95\%) \\
\hline & Sí & 52 & $0,70(0,043)$ & $1,709(233)$ & 0.089 & $0,081(-0,12-0,176)$ \\
\hline & No & 187 & $0,618(0,022)$ & & & \\
\hline & indperjuic & & & & & \\
\hline & Sí & 227 & 0,6458 & $1,65(236)$ & 0,099 & $0,155(-0,29-0,339)$ \\
\hline & No & 11 & 0,4909 & & & \\
\hline & Indagricol & & & & & \\
\hline & Sí & 21 & $0,7048(0,0664)$ & $1,077(237)$ & 0,283 & $0,07540(-0,6255-0,21336)$ \\
\hline & No & 218 & & & & \\
\hline & Indagricol & & & & & \\
\hline & Sí & 13 & $0,5692(0,0884)$ & $-0,807(237)$ & 0,421 & $-0,0759(-0,024298-0,10180)$ \\
\hline & No & 226 & & & & \\
\hline & Indagricol & & & & & \\
\hline & Sí & 119 & $0,6118(0,0289)$ & $-1,217(237)$ & 0,225 & $-0,4824(0,12629-0,02982$ \\
\hline & No & 120 & & & & \\
\hline & Indautom & & & & & \\
\hline & Sí & 15 & $0,667(0,582)$ & $0,400(237)$ & 0,69 & $0,03274(-0,12863-0,19411)$ \\
\hline & No & 224 & & & & \\
\hline
\end{tabular}


Se comparó el promedio de ISOP-CC versus el acceso a la tierra del grupo doméstico. El resultado muestra que los grupos domésticos que tienen tierra perciben en mayor medida el cambio climático (Tabla 5). Se observó que el gradiente altitudinal en el que se encuentran las tres comunidades de estudio y que representa una condición fisiográfica, relacionada con los ecosistemas presentes en cada comunidad es el factor que más influye en el índice de sensación-observación-percepción del cambio climático (ISOP-CC). A mayor altitud (comunidad alta) menor percepción del cambio climático y a menor altitud (comunidad baja) mayor percepción del cambio climático. De acuerdo con el modelo entre cada nivel de cuenca de manera ascendente hay 18,9\% menor percepción de cambio climático (Tabla 5).

Para medir la altitud, ésta se dividió en dos variables Dummy para medir el impacto de ISOP-CC a nivel de cuenca. En el nivel más alto de la cuenca, que corresponde a la comunidad de San Andrés Hueyacatitla, el 59\% de las mujeres percibió el cambio climático y el $41 \%$ no. En el nivel medio que corresponde a la comunidad de la cabecera municipal de San Salvador el Verde el 68\% de mujeres percibió cambio climático y el 32\% no. En el nivel bajo que corresponde a la comunidad de Santa Cruz Analco, $72 \%$ de las mujeres percibió el cambio climático y el $28 \%$ no. El análisis de varianza (ANOVA) demuestra que hay evidencia estadística significativa con base en la altitud de las tres comunidades de estudio $(\mathrm{F}=4,25 ; \mathrm{gl}=2 ; p=0,015)$. Este fue el principal predictor del índice de sensación-observación-per- cepción del cambio climático (ISOP-CC) entre las mujeres (Tabla 5).

Observamos diferencias significativas en la percepción del cambio climático entre la comunidad uno (baja) y tres (alta) 18,3\% más percepción de cambios. Sin embargo, entre la comunidad 1 (baja) y 2 (media) no hay diferencias significativas en la percepción del cambio climático (Tabla 5).

\subsection{Resultado del modelo de regresión}

El coeficiente de correlación de Pearson fue de $(0,293 ; p=0,086)$. El coeficiente de predicción o de determinación es de $0,054(0,29615)$ y un estadístico de Durbin Watson de 2 desviaciones estándar. La tabla de análisis de varianza (ANOVA) (Tabla 6) muestra que la suma de cuadrados de la regresión tiene un valor $\mathrm{F}=2,68$ veces superior al de la suma de cuadrados de los residuales $(p=0,008)$.

Finalmente, los coeficientes tipificados o estandarizados muestran que la ubicación de la Comunidad 3, que corresponde a San Andrés Hueyacatitla, que se relaciona con la mayor altitud a la cual se encuentra y su cercanía al bosque, es el predictor más importante: Comunidad $3(\mathrm{~B}=-0,126 ; p=0,002)$. Seguido del indicador de tenencia de la tierra del grupo doméstico $\mathrm{B}=(0,087 ; p=0,070)$, el indicador de perjuicio por el cambio climático $(\mathrm{B}=0,168$; $p=0,070)$ y el indicador de actividad primaria agrícola del esposo $(\mathrm{B}=-0,069 ; p=0,083)$ (Tabla 6$)$.

Tabla 6. Modelo de Regresión.

\begin{tabular}{|c|c|c|c|c|c|c|c|}
\hline \multicolumn{3}{|c|}{$\mathbf{R}$} & \multicolumn{2}{|l|}{ R Cuadrado } & R Ajustado & \multicolumn{2}{|c|}{$\begin{array}{c}\text { Error estándar de la } \\
\text { estimación }\end{array}$} \\
\hline \multicolumn{3}{|c|}{0,272} & 0,074 & \multicolumn{2}{|r|}{0,058} & \multicolumn{2}{|c|}{0,29548} \\
\hline \multicolumn{8}{|c|}{ ANOVA } \\
\hline Modelo & & Suma de cuadı & dos & GI & Media cuadrática & $\mathbf{F}$ & $p$ \\
\hline Regresión & & 1,622 & & 4 & 0,405 & 4,644 & 0,001 \\
\hline Residuo & & 20,343 & & 233 & 4,644 & & \\
\hline Total & & 21,964 & & 237 & 0,087 & & \\
\hline
\end{tabular}

Variable dependiente ISOP-CC

Predictores (constante), Indtienetierra, indperjuiciocam, Altitud de la comunidad, Indagricola3

\begin{tabular}{|c|c|c|c|c|c|}
\hline Modelo & B & Desv. Error & Coeficientes estandarizados Beta & $\mathbf{t}$ & $p$ \\
\hline Constante & 0,674 & 0,106 & & 6,386 & 0 \\
\hline Altitud & $-0,07$ & 0,022 & $-0,2$ & $-3,133$ & 0,002 \\
\hline Indagricola3 & $-0,068$ & 0,039 & $-0,112$ & $-1,752$ & 0,081 \\
\hline Indperjuiciocam & 0,152 & 0,092 & 0,105 & 1,658 & 0,099 \\
\hline Indtienetierra & 0,084 & 0,047 & 0,113 & 1,769 & 0,078 \\
\hline
\end{tabular}

Variable dependiente ISOP-CC 


\section{Discusión}

La construcción del índice de sensación-observación-percepción del cambio climático de las mujeres rurales de tres comunidades pertenecientes a la cuenca alta del río Atoyac, basado en el cambio actual de las variaciones climáticas, como temperatura, precipitación, fenómenos meteorológicos como vientos, heladas y granizadas, las afectaciones posteriores en términos del aumento de plagas, perjuicios en la producción agrícola, la salud y la tenencia de la tierra del grupo doméstico es importante porque permite comprender los impactos in situ del cambio climático e identificar prácticas de gestión y alternativas para los y las campesinas con miras a realizar estrategias locales de adaptación ante este fenómeno. En este estudio se analizaron las sensaciones, observaciones y percepciones de las mujeres rurales encuestadas a lo largo del gradiente altitudinal en la cuenca alta del municipio de San Salvador el Verde en la Sierra Nevada de Puebla. El empleo de la variable altitudinal coincide con (Basannagari \& Kala, 2013) quienes analizaron mediante la percepción de los entrevistados en el gradiente altitudinal los indicadores de los efectos del cambio climático en el cultivo de manzana en el distrito Kinnaur de Himachal Pradesh en Himayala, India.

En este estudio observamos que la sensación, observación y percepción del cambio climático está influenciada por el indicador de perjuicio sufrido por las entrevistadas y atribuido al cambio climático lo que coincide con Erwin (2009) quien menciona que los factores más importantes que influyen en la percepción del cambio climático y sus potenciales implicancias son el conocimiento y la disponibilidad de la información respecto a sus efectos a nivel local y la existencia de otros efectos de amenaza cuyas relaciones de causa efecto en el ecosistema resulten más evidentes que aquellos derivados del cambio climático (Erwin, 2009). Esto también coincide con lo encontrado por Uribe et al. (2017) en la región de Tarapacá donde las percepciones acerca del cambio climático responden justamente a estos factores, influenciando la consideración o desconsideración del cambio climático en instrumentos de planeación regional.

En general, las mujeres rurales de la Sierra Nevada detectan con precisión las tendencias locales de temperatura en los últimos años en comparación con los registros instrumentales de la Comisión Nacional del Agua (Conagua) en la estación de San Martín Texmelucan, Puebla. Sin embargo, en el indicador de precipitación no se detectan con precisión las tendencias locales en cuanto al volumen de lluvias, debido a que los cambios se asocian a cambios en las estaciones del año, es decir se han recorrido los meses del inicio de las lluvias, pero en el volumen de lluvias casi no ha variado de acuerdo con los registros instrumentales de la Comisión Nacional del Agua (Conagua) en la estación de San Martín Texmelucan, Puebla. Ello explica la dificultad para que las entrevistadas perciban acertadamente sí existe más o menos cantidad de lluvia.

$\mathrm{A} 1$ respecto, las entrevistadas comentan que en sus grupos domésticos han realizado ajustes en el calendario agrícola tradicional en la siembra del maíz, lo que coincide con lo encontrado por Cruz López (2011) quien trabajó la cosmovisión de los pobladores mazahuas del norte del estado de México en relación al cultivo de maíz y los cambios en el patrón anual de lluvias, lo que ha dado lugar a una dicotomía entre el tiempo meteorológico, el tiempo ritual y el tiempo de labor agrícola a la cual los pobladores se están intentando acostumbrar; a todo esto se le suma la notable disminución, año con año, de la producción agrícola y sus consecuencias económicas.

También encontramos que la intensidad de la percepción del cambio climático muestra una correlación inversamente significativa con el gradiente altitudinal de las comunidades de estudio. Esto coincide con lo reportado por Shijin \& Dahe (2015) en su estudio titulado "Perspectivas de los habitantes de las montañas sobre el cambio climático, y sus impactos y adaptación basados en el análisis de las características temporales y espaciales: un estudio de caso del Monte Yulong Snow, meseta tibetana del sudeste", quienes mencionan que el gradiente de altitud determina las condiciones naturales y la estructura socioeconómica de la vida y la producción de los residentes. Sin embargo, en lo que respecta a la edad de las entrevistadas en este estudio no encontramos diferencias significativas en el índice de sensación-observación-percepción del cambio climático, mientras que estos autores reportan que en su estudio la edad determina la profundidad de la experiencia pasada del cambio climático y la actitud hacia las ideas realistas.

Asimismo, con base en los años que han transcurrido desde que comenzaron a observar el cambio climático, la mayoría de las mujeres entrevistadas han percibido un incremento de la temperatura con una tasa del 0,91 en el periodo 1997-2011, que contempla un rango de 20 a 7 años, que abarca de 4 a 
6 años, seguidas de las que lo han percibido en el periodo 2015-2016 que contempla un rango de 3 a 2 años con una tasa de 0,87 y finalmente las que lo han percibido en el período 2012-2014 que abarca una diferencia de 4 a 6 años a la fecha con una tasa de 0,84 (Gutiérrez et al., 2019).

Tampoco, se encontró correlación estadísticamente significativa entre el índice de percepción observación y sensación de las entrevistadas y el ingreso per cápita de los grupos domésticos a los que pertenecen. Esto a su vez difiere de lo reportado por Shijin \& Dahe (2015) donde los ingresos de los residentes también fueron un factor clave que afecta su capacidad de adaptarse a los impactos del cambio climático y manifiestan una buena relación lineal con la intensidad de la actitud, es decir, es más probable que los hogares se adapten al cambio climático percibido. A diferencia de lo encontrado en este estudio, estos autores afirman que, con el aumento de los ingresos, las habilidades adaptativas de los residentes aumentan gradualmente, y sus actitudes hacia el ajuste de la estructura agrícola, la construcción de embalses de incautación y la búsqueda de una compensación del gobierno lentamente se alteró a su vez.

Las mujeres rurales entrevistadas reportan que la producción agrícola (maíz, frijol, frutales, flores y hortalizas) se ve afectada de manera adversa por las crecientes temperaturas e irregularidades en la pluviosidad, reduciendo la producción agrícola y en ocasiones la pérdida total de los cultivos. Adicionalmente, reportan la disminución de agua para riego procedente de las numerosas fuentes pluviales lo que puede estar relacionado con la disminución y desaparición de seis zonas glaciares del volcán Iztaccíhuatl (5.240 m s.n.m.), de las once que llegó a poseer y que tiene una gran importancia estratégica por la red hidrográfica que alimenta, así como por la superficie de coníferas que posee (Manzanilla-Quiñones, 2019). Lo anteriormente expuesto coincide con lo encontrado por Vandermolen (2011) en su estudio sobre las "Percepciones de cambio climático y estrategias de adaptación en las comunidades agrícolas Cotacachi”.

\section{Conclusiones}

Con base en los datos obtenidos a partir una medición cuantitativa hacia las sensaciones, observaciones y percepciones locales sobre ciertos indicadores ambientales, se considera viable identificar mecanismos manifiestos, para modelarlos estadísti- camente y poder predecir sus efectos frente a escenarios futuros de cambio climático.

La aportación metodológica de este estudio, con base en el componente socioambiental y el enfoque cuantitativo es precisamente la construcción y validación del índice de sensación-observación-percepción del cambio climático (ISOP-CC), como un instrumento que describe y caracteriza la preocupación local de las mujeres rurales acerca del entorno natural con el que llevan a cabo sus actividades cotidianas, lo que permite reconocer su perspectiva como agentes clave incluirlas de manera activa, dinámica y contextual para conferirle a sus referentes una visión integradora sobre las causas y consecuencias del cambio climático y de cómo abordarlas.

Este estudio busca servir como testimonio de los cambios que las mujeres perciben de su entorno, y que son consecuencia del cambio climático, concepto que para muchas ( $40 \%$ de la cuenca alta) no existe como tal, pero que es vivido como generador de incertidumbre sobre lo que ocurre en el ambiente. Se destaca que conocer e incluir las sensaciones, observaciones y percepciones del cambio climático de las mujeres rurales a nivel local es clave para las estrategias de desarrollo sostenible, porque permite dirigir proyectos o esfuerzos para el manejo de los recursos naturales acorde con los contextos políticos, económicos, sociales y ambientales, tomando en cuenta las referencias locales. Es deseable que las estrategias de adaptación al cambio climático se construyan con base en procesos participativos más incluyentes y equitativos, esenciales para llevar a cabo una utilización sostenible de los recursos naturales y mantener el bienestar de los campesinos y campesinas de las comunidades rurales. De esta manera se muestra como el índice de sensación, observación y percepción del cambio climático se constituye en un factor fundamental para entender las formas de adaptación y el cambio de prácticas productivas a nivel local.

E1 ISOPS-CC fue construido a partir de la percepción, observación y sensaciones de las mujeres rurales, sobre los indicadores ambientales y de desarrollo sostenible que pueden fortalecer decisiones informadas por parte de los tomadores de decisiones y encargados de la política ambiental. Como en el caso de la Secretaría de Bienestar y la Secretaría de Medio Ambiente y Recursos Naturales (Semarnat) a través de la Comisión Nacional Forestal (Conafor), así como la participación ciudadana, para impulsar a las comunidades de estu- 
dio de la Sierra Nevada de Puebla hacia un manejo integral del territorio, el desarrollo sostenible bajo en emisiones de gases de efecto invernadero y una agricultura climáticamente inteligente.

Las mujeres pertenecientes a San Andrés Huayacatitla, que corresponde a la comunidad que se encuentra a mayor gradiente altitudinal, son las que menos perciben los impactos del cambio climático, esto se debe a que la comunidad alta cuenta con mayor capital natural que corresponde al recurso bosque y donde se realiza manejo forestal sustentable, lo que coincide con la teoría ecosistémica que menciona que a mayor altitud menor vulnerabilidad socioambiental. Sin embargo, el hecho de que en esta comunidad se reporta el mayor número de habitantes, aunado a que más del $40 \%$ de las mujeres entrevistadas no perciba los impactos del cambio climático, son hallazgos que alertan sobre las condiciones sociales actuales que deben considerarse como parte de la evaluación de vulnerabilidad ante el cambio climático o deterioro ambiental en un futuro. La ausencia de referentes conceptuales entre la población local, hace que se omitan acciones cotidianas que contribuyen al cambio climático.

A manera de recomendación, es importante sugerir que las instituciones gubernamentales y civiles presentes en el área lleven a cabo charlas y capacitaciones referentes al cambio climático, con las mujeres y con la población en general, dado que los conceptos respectivos, suelen ser mal utilizados, pues con frecuencia se emplea el término de cambio climático, sin tener claridad en las fuentes de origen ni sus implicaciones.

Con base en los factores socioeconómicos que fueron analizados en este estudio, no se encontró asociación entre la educación de las mujeres, medida mediante los años de estudio, y sus actividades económicas primarias y secundarias. Tampoco se presenta correlación significativa entre la actividad económica principal del esposo y el índice de

\section{Referencias}

Adger, W. N, \& Kelly, P. M. (1999). Social Vulnerability to Climate Change and the Architecture of Entitlements. Mitigation and Adaptation Strategies for Global Change, 4(3), 253-266. https://doi.org/10.1023/A:1009601904210

Aznar-Casanova, J. A. (2018). Psicología de la percepción visual. Universidad de Barcelona. http://www.ub.edu/pa1/ percepción observación y sensación de las mujeres rurales estudiadas. Sin embargo, se presenta correlación significativa entre las mujeres que reportan haber sufrido perjuicios por el cambio climático y la tenencia de la tierra del grupo doméstico con el índice de sensación-observación-percepción del cambio climático.

En la región de estudio que forma parte del Área Natural Protegida Parque Nacional Izta-Popo, es necesario que se lleven a cabo programas de monitoreo forestal, ambiental y climático continuo y la sistematización de sus posibles implicaciones.

Los registros climáticos instrumentales, que se obtienen de las estaciones meteorológicas a cargo de la Comisión Nacional del Agua (Conagua), resultan esenciales para detectar patrones a largo plazo y ayudar al diseño de estrategias de manejo sustentable de los recursos naturales que contribuyan a la mitigación y adaptación del cambio climático a nivel local. La falta de certeza sobre los probables efectos del cambio climático en la Sierra Nevada de Puebla se debe principalmente a la escasez de investigaciones que permitan la modelación de los efectos e impactos del fenómeno a futuro.

\section{Agradecimientos}

Al Programa de Investigación en Cambio Climático de la Universidad Nacional Autónoma de México (PICC-UNAM) por el apoyo financiero al proyecto "Elaboración de la estrategia local de adaptación al cambio climático con enfoque de género en el municipio de San Salvador el Verde en la Sierra Nevada de Puebla". Al Consejo Nacional de Ciencia y Tecnología (CONACYT) por su apoyo al Proyecto Cátedras 1670. Estrategias de adaptación al cambio climático por localidades campesinas de la Sierra Nevada de Puebla. A las autoridades municipales y ejidales, a las mujeres que amablemente participaron en este proyecto.

Basannagari, B., \& Kala, C.P. (2013) Climate change and apple farming in Indian Himalayas: A study of local perceptions and responses. PLOS ONE 8(10): e77976. https://doi.org/10.1371/ journal.pone. 0077976

Bertoni, M., \& López, M. J. (2010). Percepciones sociales ambientales: Valores y actitudes hacia la conservación de la reserva de biosfera "Parque 
Atlántico Mar Chiquita", Argentina. Estudios y Perspectivas en Turismo, 19(5), 835-849.

Cegarra, N. (2019). Metodología CEPAL para construir y sostener indicadores ambientales, cambio climático y desastres [Diapositiva de PowerPoint]. Comisión Económica para América Latina y el Caribe [CEPAL]. https:// www.cepal.org/sites/default/files/presentations/5-construccion-indicadores-cepal_0.pdf

Cochran, W. G. (2000). Técnicas de muestreo. Compañía Editorial Continental.

Consejo Nacional de Evaluación de la Política de Desarrollo Social [CONEVAL]. (2019). Guía para el establecimiento y cálculo de lineas base y metas. Obtenido de https:// www.coneval.org.mx/coordinacion/Documents/monitoreo/metodologia/guia_lineas base_metas.pdf

Consejo Territorial de Planeación Distrital. (2020). Concepto de índice e indicador. Obtenido de https://www.ctpdbogota.org/concepto-de-indice-e-indicador/

Cruz López, M. (2011). Comparación del ciclo agrícola actual con el de hace unos diez años en San Juan Jalpa municipio San Felipe del Progreso Estado de México: Evidencia de adaptación al cambio climático. Ra Ximhai, 7(1), 95-106.

Demeritt, D. (2001). Being constructive about nature. In N. Castree \& B. Braun (Eds.) Social Nature: Theory, Practice, and Politics. (pp-. 22-42). Blackwell.

Deressa, T., Hassan, R., Ringle, C., Alemu, T., \& Yesuf, M. (2009). Determinants of farmer's choice of adaptation methods to climate change in the Nile Basin of Ethiopia. Global Environmental Change, 19(2), 248-255. https:// doi.org/10.1016/j.gloenvcha.2009.01.002

Dietz, A. J., Geest, K. van der, \& Obeng, F. (2013). Local perceptions of development and change in Northern Ghana. In J. A. Yaro (Ed.) Rural development in Northern Ghana. (17-36). Nova

Donnelly, D. V., Berg, W. P., \& Fiske, D. M. (2006). The effect of the direction of gaze on the kinematics of the squat exercise. Journal of strength and conditioning research, 20(1), 145-150. https://doi.org/10.1519/R-16434.1
Duarte Cueva, F. (2014). Efectos del cambio climático en la economía, el comercio internacional y la estrategia empresarial. Contabilidad y $\mathrm{Ne}$ gocios, 9(18), 75-98.

Erwin, K. L. (2009). Wetlands and global climate change: the role of wetland restoration in a changing world. Wetlands Ecology and $\mathrm{Ma}$ nagement, 17, 71-84. https://doi.org/10.1007/ s11273-008-9119-1

Forsyth, T. (2003). Critical Political Ecology: the Politics of Environmental Science. Routledge.

Fuentes Flores, N. A., \& Brugués, A. (2017). Impactos Económicos del cambio climático con base en un enfoque sistémico. Revista de Economía, 34(89), 9-41. https://doi.org/10.33937/ reveco. 2017.86

González-Pérez, E., Ortega-Escobar, H. M., Yáñez-Morales, M. J., \& Rodríguez-Guillen, A. (2019). Diagnóstico de indicadores de calidad físico-química del agua en afluentes del río Atoyac. Tecnología y Ciencias del Agua, 10(1), 1-28. https://doi.org/10.24850/j-tyca-2019-01-02

Grupo Intergubernamental de Expertos sobre el Cambio Climático [IPCC]. (2007). Cambio climático 2007: Informe de sintesis. Contribución de los Grupos de trabajo I, II y III al Cuarto Informe de evaluación del Grupo Intergubernamental de Expertos sobre el Cambio Climático. Equipo de redacción principal: Pachauri, R.K., \& Reisinger, A. (directores de la publicación)]. IPCC.

Gutiérrez Villalpando, V., Martínez Corona, B., \& Salvatierra Izaba, B. (2019). Género y Vulnerabilidad del agua ante la variabilidad climática en la Sierra Nevada de Puebla. En D. A. Fabre Platas, I. Ortiz Sánchez, \& G. Busso (Coords.), Agua: Territorialidades y dimensionalidades de análisis (pp. 463-483). Serie Mano Vuelta. Editorial Resistencia SA de CV

Hodgdon, B. D., Hughell, D., Ramos, V. H., \& McNab, R. B. (2013). Deforestation Trends in the Maya Biosphere Reserve, Guatemala (2000 2013). Rainforest Alliance, Wildlife Conservation Society, Consejo Nacional de Áreas Protegidas, Guatemala. 
Howe, P., Markowitz, E., Lee, T. M., Ko, C. Y., \& Leiserowitz, A. (2013). Global perceptions of local temperature change. Nature Climate Change, 3, 352-356. https://doi.org/10.1038/nclimate1768

Ibáñez Pérez, R. M. (2012). Indicadores de sustentabilidad: utilidad y limitaciones. Teoría y Praxis, 11, 102-126.

Infante Ramírez, K. D., \& Arce Ibarra, A. M. (2015). Percepción local de los servicios ecológicos y de bienestar de la selva de la zona maya en Quintana Roo, México. Repositorio UNAM: Investigaciones Geográficas, 86, 67-81. https:// doi.org/10.14350/rig.36593

Instituto Nacional de Ecología y Cambio Climático [INECC]. (2020). Construcción de esquemas de monitoreo y evaluación de la adaptación en México para la formulación de politicas públicas basadas en evidencia. INECC. https://www.gob.mx/ cms/uploads/attachment/file/533194/Libro_sintesis_Construccion_de_Esquemas_de_MyE.pdf

Lazos Chavero, E., \& Pare Ouellet, L. (2016). Miradas indigenas sobre una naturaleza entristecida. UNAM.

Manzanilla-Quiñones, U., Aguirre-Calderón, O. A., Jiménez-Pérez, J., Treviño-Garza, E. J., y Yerena-Yamallel, J. I. (2019). Distribución actual y futura del bosque subalpino de Pinus hartwegii Lindl en el Eje Neovolcánico Transversal. $M a$ dera y bosques, 25(2), e2521804. https://doi. org/10.21829/myb.2019.2521804

Martínez-Austria, P. F., \& Patiño-Gómez, C. (2012). Efectos del cambio climático en la disponibilidad de agua en México. Tecnología y ciencias del agua, 3(1), 5-20.

Mayo Mendoza, M. (2019). Conocimiento tradicional, ¿Una alternativa al cambio climático?. Kuxulkab', 25(51), 41-47. https://doi. org/10.19136/kuxulkab.a25n51.2901

Millán Escriche, M. (2004). La geografía de la percepción: una metodología de análisis para el desarrollo rural. Papeles De Geografia, 40, 133-149.

Norma Oficial Mexicana NOM-161-SEMARNAT-2011, Que establece los criterios para clasificar a los Residuos de Manejo Especial y de- terminar cuáles están sujetos a Plan de Manejo; el listado de los mismos, el procedimiento para la inclusión o exclusión a dicho listado; así como los elementos y procedimientos para la formulación de los planes de manejo. 23 de noviembre de 2012 (México). http://www.dof.gob.mx/nota_detalle.php? codigo $=5286505 \&$ fecha $=01 / 02 / 2013$

Oltra, C., Solà, R., Sala, R., Prades, A., \& Gamero, N. (2009). Cambio climático: percepciones y discursos públicos. Prisma Social, 2,1-23.

Organización para la Cooperación y el Desarrollo Económico [OECD]. (1993). OECD Core set of indicators for environmental performance reviews. OECD. Environmental Monographs $n^{\circ} 83$.

Orozco, I., Ramírez, A. I., \& Francés, F. (2018). Modelación de los impactos del cambio climático sobre los flujos y almacenamientos en una cuenca de alta montaña. Ingeniería del agua, 22(3), 125-139. https://doi.org/10.4995/ ia.2018.8931

Perevochtchikova, M. (2012). La evaluación del impacto ambiental y la importancia de los indicadores ambientales. Gestión y política pública, 22(2), 283-312.

Pinilla Herrera, M., Rueda, A., Pinzón, C., \& Sánchez, J. (2012). Percepciones sobre los fenómenos de variabilidad climática y cambio climático, entre campesinos del centro de Santander, Colombia. Ambiente y Desarrollo, 16(31), 25-37.

Revuelta Vaquero, B. (2019). La aportación local y regional, ante el cambio climático en México. Un balance de las políticas públicas. En Universidad Nacional Autónoma de México y Asociación Mexicana de Ciencias para el Desarrollo Regional A.C (Coeditores), Impactos ambientales, gestión de recursos naturales y turismo en el desarrollo regional. UNAM.

Roth, A. (2019). Cambio climático y políticas públicas: un abordaje desde la teoría social del riesgo. Prometeica - Revista de Filosofia y Ciencias, 18, 36-46. https://doi.org/10.24316/ prometeica.v0i18.250

Ruiz Meza, L. E. (2014). Género y percepciones sociales del riesgo y la variabilidad climática en 
la región del Soconusco, Chiapas. Alteridades, 24(47), 77-88.

Secretaría de Medio Ambiente y Recursos Naturales [Semarnat] \& Centro de Educación y Capacitación para el Desarrollo Sustentable [CECADESU]. (2016). Adaptación al cambio climático, fundamentos desde el manejo de cuencas y del proceso de fortalecimiento de capacidades. Cuadernos de divulgación ambiental. Semarnat.

Shah, R. (2004). CSD Indicators of Sustainable Development - recent developments and activities. Assessment of Sustainability Indicators (ASI): A SCOPE/UNEP/IHDP/EEA Project. ASI Workshop. Division for Sustainable Development. Department of Economic and Social Affairs, New York.

Shijin, W., \& Dahe, Q. (2015). Mountain inhabitant's perspectives on climate change, and its impacts and adaptation based on temporal and spatial characteristics analysis: a case study of Mt. Yulong Snow, Southeastern Tibetan Plateau. Environmental Hazards, 14(2), 122-136. https://doi. org/10.1080/17477891.2014.1003776

Soares, D., García, A., \& Manzano Solís, L. R. (2018). Cambio climático. Percepciones sobre manifestaciones, causas e impactos en el Distrito de Temporal Tecnificado Margaritas-Comitán, Chiapas. CIENCIA Ergo-Sum, 25(1).. https://doi.org/10.30878/ces.v25n1a1

Solís Mecalco, R. J., \& Salvatierra Izaba, B. (2012). Percepción social del cambio climá- tico en Áreas Destinadas Voluntariamente a la Conservación en comunidades indígenas de Oaxaca y Chiapas. Temas Antropológicos. Revista Cientifica de Investigaciones Regionales, 35(1), 29-53.

Spence, A., Poortinga, W., Butler, C., \& Pidgeon, N. F. (2011). Perceptions of climate change and willingness to save energy related to flood experience. Nature climate change, 1, 46-49. https://doi.org/10.1038/nclimate1059

Ulloa, A. (2011). Perspectivas culturales del clima. Universidad Nacional de Colombia, Facultad de Ciencias Humanas, Departamento de Geografía.

Uribe Rivera, D., Vera Burgos, C., Paicho, M., \& Espinoza, G. (2017). Observatorio ecosocial para el seguimiento del cambio climático en ecosistemas de altura en la región de Taracapá: Propuestas, avances y proyecciones. Diálogo Andino - Revista de Historia, Geografia y Cultura Andina, 54, 63-82.

VanderMolen, K. (2011). Percepciones de cambio climático y estrategias de adaptación en las comunidades agrícolas de Cotacachi (Debate Agrario-Rural). Ecuador Debate, 82, 145157.

Women's Environmental Network. (2010). Gender and the Climate Change Agenda: The impacts of climate change on women and public policy. World Development Movement, ActionAid, Progressio, Women's Environmental Network. 